\title{
Constraints on Saturn's tropospheric general circulation from Cassini ISS images
}

\author{
Anthony D. Del Genio ${ }^{\mathrm{a}, *}$, John M. Barbara ${ }^{\mathrm{b}}$ \\ a NASA Goddard Institute for Space Studies, 2880 Broadway, New York, NY 10025, United States \\ b ${ }^{\text {S } i g m a ~ S p a c e ~ P a r t n e r s, ~ I n s t i t u t e ~ f o r ~ S p a c e ~ S t u d i e s, ~} 2880$ Broadway, New York, NY 10025, United States
}

\section{A R T I C L E I N F O}

\section{Article history:}

Received 16 December 2011

Revised 19 March 2012

Accepted 30 March 2012

Available online 9 April 2012

\section{Keywords:}

Atmospheres, Dynamics

Meteorology

Saturn, Atmosphere

\begin{abstract}
A B S T R A C T
An automated cloud tracking algorithm is applied to Cassini Imaging Science Subsystem high-resolution apoapsis images of Saturn from 2005 and 2007 and moderate resolution images from 2011 and 2012 to define the near-global distribution of zonal winds and eddy momentum fluxes at the middle troposphere cloud level and in the upper troposphere haze. Improvements in the tracking algorithm combined with the greater feature contrast in the northern hemisphere during the approach to spring equinox allow for better rejection of erroneous wind vectors, a more objective assessment at any latitude of the quality of the mean zonal wind, and a population of winds comparable in size to that available for the much higher contrast atmosphere of Jupiter. Zonal winds at cloud level changed little between 2005 and 2007 at all latitudes sampled. Upper troposphere zonal winds derived from methane band images are $\sim 10 \mathrm{~m} \mathrm{~s}^{-1}$ weaker than cloud level winds in the cores of eastward jets and $\sim 5 \mathrm{~m} \mathrm{~s}^{-1}$ stronger on either side of the jet core, i.e., eastward jets appear to broaden with increasing altitude. In westward jet regions winds are approximately the same at both altitudes. Lateral eddy momentum fluxes are directed into eastward jet cores, including the strong equatorial jet, and away from westward jet cores and weaken with increasing altitude on the flanks of the eastward jets, consistent with the upward broadening of these jets. The conversion rate of eddy to mean zonal kinetic energy at the visible cloud level is larger in eastward jet regions $\left(5.2 \times 10^{-5} \mathrm{~m}^{2} \mathrm{~s}^{-3}\right)$ and smaller in westward jet regions $\left(1.6 \times 10^{-5} \mathrm{~m}^{2} \mathrm{~s}^{-3}\right)$ than the global mean value $\left(4.1 \times 10^{-5} \mathrm{~m}^{2} \mathrm{~s}^{-3}\right)$. Overall the results are consistent with theories that suggest that the jets and the overturning meridional circulation at cloud level on Saturn are maintained at least in part by eddies due to instabilities of the large-scale flow near and/or below the cloud level.
\end{abstract}

Published by Elsevier Inc.

\section{Introduction}

One of the primary objectives of the Cassini Orbiter mission to Saturn has been to document Saturn's general circulation and to provide observational constraints on theories advanced to explain the circulation. Prior to Cassini, Saturn was less comprehensively observed than Jupiter, but 4 years of data from the Cassini nominal mission and new data from the extended mission now allow us to place Saturn on at least an equal footing with Jupiter despite the greater observational challenges presented by Saturn's multi-layer hazes and clouds.

The history of observations of Saturn's dynamics and the evolution of our understanding of the relevant processes up through the early years of the Cassini mission is discussed in the review by Del Genio et al. (2009). Like Jupiter, Saturn's major observed dynamical feature is its series of alternating eastward and westward jets, although whether Saturn actually has westward jets or merely eastward wind minima depends on the poorly known rotation rate

\footnotetext{
* Corresponding author.

E-mail addresses: anthony.d.delgenio@nasa.gov (A.D. Del Genio),john.m.barbara@ nasa.gov (J.M. Barbara).
}

of the deep atmosphere (Desch and Kaiser, 1981; Anderson and Schubert, 2007; Read et al., 2009a,b). The past few years has seen considerable progress in numerical modeling of jovian planet atmospheres targeted at understanding possible mechanisms for the observed jet structure. The modeling efforts fall into two broad categories - those that emphasize processes occurring in shallow weather layers near the visible cloud level (e.g., Lian and Showman, 2010; Liu and Schneider, 2010), and others that explore the ramifications of processes that extend through the deep molecular hydrogen envelopes of the jovian planet atmospheres (e.g., Heimpel and Aurnou, 2007; Kaspi et al., 2009). It has often been assumed that observations of the vertical structure of the wind itself differentiate these two classes of theories. However, it is possible for shallow forcing to influence the dynamics at depth and vice versa, so knowledge of the vertical structure of the mean zonal wind provides a valuable observational constraint but must be accompanied by other constraints to identify the relevant mechanisms (Showman et al., 2006; Del Genio et al., 2009).

The Cassini Imaging Science Subsystem (ISS; Porco et al., 2004) is one of the primary sources of information available to document dynamical processes operating in Saturn's atmosphere. The narrow-angle camera (NAC) allows individual regions on Saturn 
to be imaged at high resolution near distant apoapses, making hemispheric or near-global mosaics possible, while the accompanying wide-angle camera (WAC) provides simultaneous global views required for image navigation. ISS observes Saturn from the ultraviolet to the near-infrared, including both continuum and methane band filters, which produces information on clouds and hazes from Saturn's middle troposphere to stratosphere.

ISS images of the southern hemisphere of Saturn from the Cassini approach to Saturn and from early orbits at mostly moderate resolution have been used to document the latitudinal profile of mean zonal wind and its changes since the Voyager and Hubble eras (Porco et al., 2005; Vasavada et al., 2006; Sánchez-Lavega et al., 2007; García-Melendo et al., 2009); later images provide global coverage (García-Melendo et al., 2011). These studies primarily used either a manual tracking or a one-dimensional line shifting correlation approach. Manual tracking provides the highest confidence in individual wind vectors but its sampling is limited and the results can vary due to the subjective selection of targets. Line-shifting methods using a large segment of a latitude circle allow for very high latitudinal resolution but can only provide information on mean zonal winds, while potentially being influenced by large-scale waves whose phase speeds differ from the speed of the underlying wind (e.g., Del Genio and Rossow, 1990).

Del Genio et al. (2007) applied an automated cloud tracking approach to Saturn using a two-dimensional feature correlation method. The primary advantage of this method is that it uniformly samples an entire image in small areas corresponding to one or a few individual cloud features. It thus provides a more objective estimate of the actual mean wind (to the extent that the vectors that satisfy quality criteria are not a spatially biased sample). Furthermore it allows higher order statistics such as eddy momentum fluxes to be calculated since it yields two-dimensional information on the wind field at any level (three-dimensional if multiple filters are used). Because the features tracked are two-dimensional, though, it provides lower latitudinal resolution than one-dimensional techniques, and because it is automated it is subject to erroneous estimates of the wind in areas with little albedo contrast or mostly linear features.

Del Genio et al. (2007) applied the automated method to ISS Saturn near-infrared continuum filter images from early in the Cassini nominal mission. Because of the limitations mentioned above, we were only able to retrieve reliable wind estimates at some latitudes, the most problematic regions being the latitudes of the eastward jet cores. The early images also covered only the southern hemisphere due to obscuration of the northern hemisphere by the rings, ring shadows, and lack of insolation. Nonetheless the technique was good enough to provide an order of magnitude more sampling than was possible with the Voyager images (Ingersoll et al., 1984), while also allowing for the first credible estimates of the eddy momentum flux distribution on Saturn.

In this paper we extend our results to the 2007 time period, when Cassini was placed into a series of large daytime apoapsis orbits at low phase angle designed specifically to optimize largescale imaging. These orbits give us our first views of the northern hemisphere of Saturn, permitting us to document the global wind characteristics but also giving us sufficient sampling to create regional composites of essential features of the dynamics. Additional recent images (2011-2012) allow us to track more of the previously obscured equatorial region as well. We also describe an improvement in our cloud tracking scheme that allows us to sample more of the eastward jet core regions with confidence, reduce uncertainties in our estimates of eddy momentum flux, and perform the first fully automated tracking of individual cloud feature areas in Saturn methane band images. Section 2 describes the data and methods used as well as several sensitivity tests of the algorithm. Section 3 documents the mean wind and eddy momentum flux distribution at a continuum wavelength, while Section 4 compares these to similar estimates at a methane band wavelength. Section 5 discusses the implications of our results for dynamical theories of jovian planet jet maintenance and the meridional overturning circulation.

\section{Data and methods}

\subsection{Data selection and processing}

The primary dataset used consists of ISS NAC continuum band (CB2; $750 \mathrm{~nm})$ high-resolution $\left(\sim 17-23 \mathrm{~km} \mathrm{pixel}^{-1}\right)$ images acquired on one early orbit in 2005 (Rev 3 ) and 3 of the later large dayside apoapsis orbits in 2007 (Revs 48, 49, 52). During Rev 48 , limitations on data volume due to competition with other instruments for tape recorder space required $2 \times 2$ pixel summing, reducing the effective resolution. A total of 360 CB2 images taken from 28 mosaic pairs separated by approximately one Saturn rotation were analyzed. We also used methane band images taken with the ISS NAC MT2 filter $(727 \mathrm{~nm})$ simultaneously with the CB2 images on Revs 3 and 52. The MT2 images on the latter orbit were $2 \times 2$ summed. A total of 180 MT2 images from 16 mosaic pairs were analyzed. There are no data between $6^{\circ} \mathrm{S}$ and $18^{\circ} \mathrm{N}$ during these time periods due either to ring shadow or obscuration or image sampling. However, 20 lower resolution (103-172 $\mathrm{km} \mathrm{pixel}^{-1}$ ) WAC CB2s acquired during the extended mission in 2011-2012 (Revs 145 and 159-161), by which time low northern latitudes were illuminated, have allowed us to extend northern hemisphere coverage down to $5.5^{\circ} \mathrm{N}$. The details of each imaging period are given in Table 1. The subsolar latitude for the NAC images ranged from $22.6^{\circ} \mathrm{S}$ during Rev 3, to $9.9^{\circ} \mathrm{S}$ during Rev 52. For the recent WACs the subsolar latitude ranged from $8.4^{\circ} \mathrm{N}$ during Rev 145 to $13.2^{\circ} \mathrm{N}$ during Rev 161 .

Image calibration, photometric correction, and navigation procedures are described fully in Del Genio et al. (2007). Briefly, we use Cassini Imaging Science Subsystem CALibration (CISSCAL) software (Porco et al., 2004; see also R. West, 2005, unpublished manuscript, available from the Planetary Data System) to subtract dark current and bias, divide by a flat field, correct for nonlinearity and dust rings, adjust to absolute calibrations when available, and convert to $I / F$ units using the insolation at Saturn's distance as a reference. A Minnaert function is then applied to partly correct for large-scale illumination gradients. Navigation of NACs exploits near-simultaneous WACs to locate the limb with sub-pixel precision, match approximate predicted pointing from binary C-kernels to the limb curve with a least-squares fit, and then apply a small WAC-NAC boresight correction. Navigated images are finally mapped into a cylindrical (rectangular) projection in the planetocentric latitude system.

Near-infrared images of Saturn are affected both by the optically thick ( $\tau \sim 10$ ) upper troposphere haze that is estimated to extend from $\sim 100$ to 400 mbar and by the ammonia cloud deck that begins in the vicinity of $\sim 1$ bar (Karkoschka and Tomasko, 2005; Pérez-Hoyos et al., 2005). Thus temporal and spatial variations in the thickness of the tropospheric haze affect the level to which CB2 sees. Fig. 1 (left) shows a typical 2007 WAC CB2 image of Saturn. The two hemispheres have very different appearances, the northern hemisphere being darker but having much greater small-scale feature contrast than the southern hemisphere. Fletcher et al. (2011) find a factor of 1.5-2.0 seasonal difference in the $5 \mu \mathrm{m}$ opacity of the tropospheric haze, with opacity being lower in the winter (northern) hemisphere. The thinner northern haze apparently allows CB2 images to see deeper into Saturn's atmosphere there. Fig. 2 (solid curves) shows that mean $I / F$ for the features we tracked in 2007 was lower in the northern hemisphere 
Table 1

Details of the ISS images used to track Saturn winds in this paper.

\begin{tabular}{|c|c|c|c|c|}
\hline Dates & Filters & \# of image pairs & Resolution (km pixel ${ }^{-1}$ ) & Latitude range \\
\hline February 5-8, 2005 & CB2, MT2 & 60 & $17-19$ & $6^{\circ} \mathrm{S}-66^{\circ} \mathrm{S}$ \\
\hline August 2-3, 2007 & $\mathrm{CB} 2$ & 72 & 45 & $18^{\circ} \mathrm{N}-46^{\circ} \mathrm{N}, 6^{\circ} \mathrm{S}-40.5^{\circ} \mathrm{S}$ \\
\hline August 12-13, 2007 & $\mathrm{CB} 2$ & 18 & $17-19$ & $6^{\circ} \mathrm{S}-66^{\circ} \mathrm{S}$ \\
\hline November 6-7, 2007 & CB2, МT2 & 30 & 17-19 (CB2) 34-38 (MT2) & $18^{\circ} \mathrm{N}-60^{\circ} \mathrm{N}$ \\
\hline February 23-24, 2011 & $\mathrm{CB} 2$ & 2 & $103-116$ & $5.5^{\circ} \mathrm{N}-18^{\circ} \mathrm{N}$ \\
\hline January 9-14, 19, 23, 2012 & $\mathrm{CB} 2$ & 7 & $130-172$ & $5.5^{\circ} \mathrm{N}-18^{\circ} \mathrm{N}$ \\
\hline February 16, 2012 & $\mathrm{CB} 2$ & 1 & 119 & $5.5^{\circ} \mathrm{N}-18^{\circ} \mathrm{N}$ \\
\hline
\end{tabular}

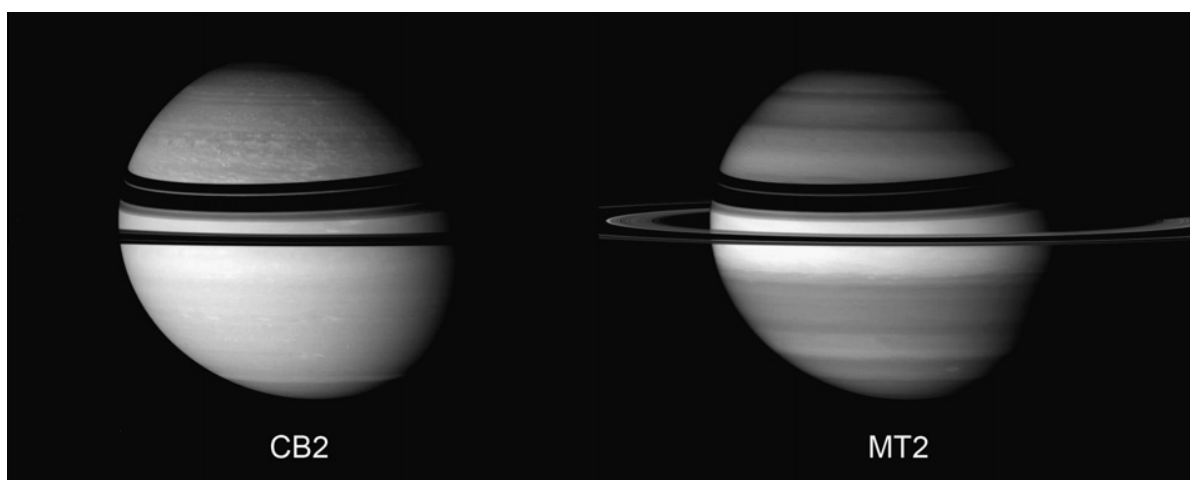

Fig. 1. ISS CB2 (left) and MT2 (right) Rev 52 images of Saturn taken on November 6, 2007.
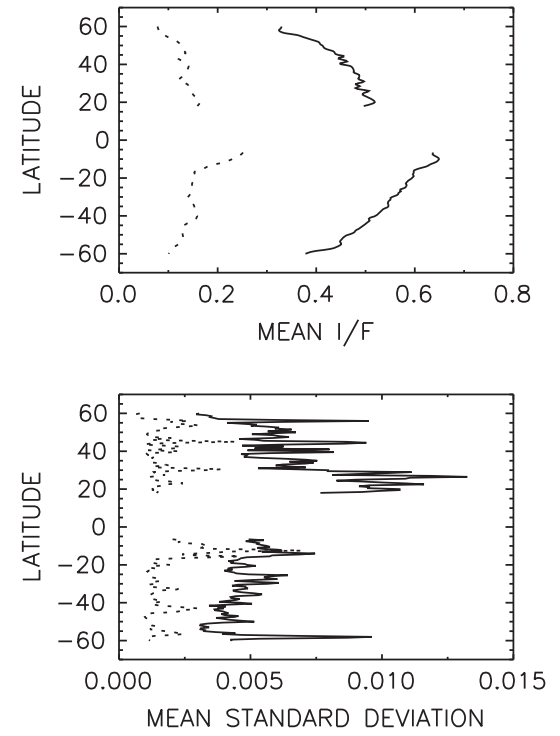

Fig. 2. Upper panel: Mean $I / F$ vs. planetocentric latitude for tracking target areas in Rev 52 CB2 (solid) and MT2 (dotted) WAC images. Lower panel: Mean standard deviation of $I / F$ within individual tracking boxes vs. latitude for the same images.

than the southern hemisphere, while the mean standard deviation of $I / F$ within our tracking target boxes (see Section 2.2) was significantly larger in the northern hemisphere than at equivalent latitudes in the southern hemisphere. (Note that Fig. 2 uses WAC images, which show both hemispheres in both the CB2 and MT2 filters simultaneously. The standard deviations for the NACs that are actually used for most of our tracking are larger than those shown in Fig. 2 for the WACs, but the sense of the hemispheric difference is similar.) This makes CB2 tracking much easier at northern latitudes, especially in the vicinity of the eastward jets where relatively few discrete features exist.

Fig. 1 (right) shows a near-simultaneous MT2 image of Saturn. Regardless of haze optical thickness variations, MT2 seeing is limited to the upper troposphere by methane absorption. MT2 images are more zonally uniform in appearance with relatively few discrete features by comparison to CB2 images, making automated tracking significantly more challenging. Fig. 2 (dotted curves) shows that relative to $\mathrm{CB} 2$ images taken at the same time, MT2 images are not surprisingly much darker, and the northern hemisphere is only slightly darker than the southern hemisphere. The standard deviations of MT2 I/F values are much more hemispherically symmetric than those for CB2, confirming that with the exception of the occasional deep convective storm, CB2 and MT2 represent mostly independent information from two different altitudes (the middle troposphere ammonia cloud and the upper troposphere haze).

\subsection{Cloud tracking algorithm}

The automated cloud tracking algorithm we use is described in detail in Del Genio et al. (2007). Briefly, evenly spaced $\sim 2^{\circ}$ longitude by $\sim 1^{\circ}$ latitude $(100 \times 50$ pixels $)$ target boxes are defined in the first image of a pair. Each target box is displaced by $0.5^{\circ}$ with respect to the previous one. A large area $(1200 \times 150$ pixels $)$ of the second image, centered on the original target location, is searched to find the location of the maximum correlation, and the wind vector is calculated from the displacement divided by the time interval between the images. Wind vectors with maximum correlation coefficient $<0.5$ are rejected, as are vectors for which either the zonal or meridional wind speed exceeds one standard deviation of the mean speed at that latitude. This eliminates many spurious vectors associated with significant shape changes, large-scale brightness gradients not removed by image processing, and periodic feature morphologies that produce multiple correlation maxima (see, e.g., Fig. 3 of Rossow et al. (1990)). MT2 wind speeds have considerable scatter, so we use the CB2 mean at each latitude instead with the MT2 standard deviations as our window for accepting MT2 wind vectors.

This procedure works well for CB2 images over much of Saturn, but leaves noticeable gaps in the vicinity of the eastward jets, especially in the lower contrast southern hemisphere (see, e.g., Fig. 4 of 
Del Genio et al. (2007)). It also performs poorly for most latitudes in MT2 images. Fig. 3 shows the distribution of $\mathrm{CB} 2$ wind vectors for two extreme cases: A latitude with well-defined discrete cloud features for which tracking is accurate based on visual inspection of many individual cases $\left(59.5^{\circ} \mathrm{N}\right)$, and another with poor contrast and more curvilinear features where the tracking algorithm obviously fails $\left(55.5^{\circ} \mathrm{S}\right)$. Other latitudes exhibit features of both distributions, with a central unimodal distribution but also randomly distributed outlier vectors (see Section 5).

We therefore experimented with an additional quality criterion that (a) identifies the wind speed at each latitude at which the distribution drops to $1 \%$ of the number of vectors at the mode value, and (b) rejects wind speed values outside the $1 \%$ bounds. Visual inspection of images corresponding to a random subset of the rejected vectors suggests that most of them (59 out of 64) are indeed artifacts. We also tested a more stringent cutoff of $10 \%$, but we found that 11 of 15 randomly selected vectors between the $1 \%$ and $10 \%$ cutoff points were good vectors. We finally require that at least $50 \%$ of the wind speed values at each latitude fall within the $\pm 1 \%$ points of the distribution for us to accept the mean wind and eddy momentum flux values for the entire latitude band $\left(55.5^{\circ} \mathrm{S}\right.$ being a case that fails the test). This allows us to extend our results to the cores of several eastward jets (or at least close to them) and to produce credible MT2 wind and eddy flux estimates at many latitudes. As can be seen from Fig. 3, even the well-behaved distributions are not perfectly symmetric about the mode value, so we tried defining the $1 \%$ point of the distribution in two ways: At equal distances from the histogram peak to the first $1 \%$ value encountered on either side, and using the actual $1 \%$ points of the distribution on the faster and slower sides of the peak. Of 47 visually inspected wind vectors that failed the first test but passed the second test, $87 \%$ were either clearly erroneous or uncertain, so we adopted the simpler first approach in the results presented in the following sections.

We conducted two other sensitivity tests to evaluate our ability to accurately determine the mean wind speed at jet cores. Our $1^{\circ}$ latitudinal width target box might, for example, smooth jet peaks and create errors due to wind shear across the box. We therefore
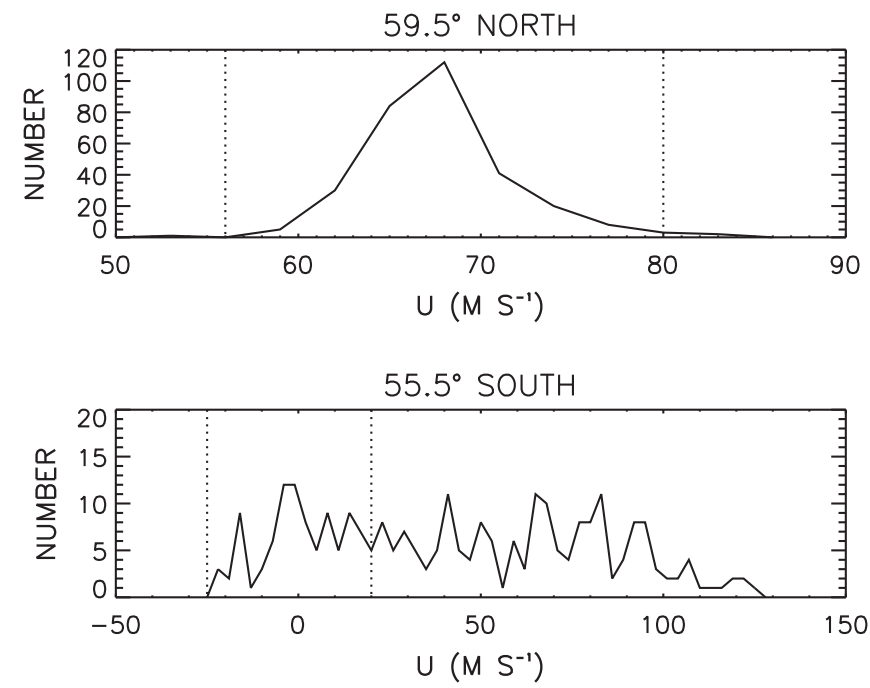

Fig. 3. Probability density function of zonal wind speeds at $59.5^{\circ} \mathrm{N}$ (upper) and $55.5^{\circ} \mathrm{S}$ (lower) planetocentric latitude. The vertical dotted lines indicate the wind speed at which the number of vectors first decreases on either side to $1 \%$ of the number of vectors at the mode value of the distribution (the resulting cutoff being applied symmetrically on both sides of the mode value). Wind speeds outside this range are discarded from the calculation of the zonal mean and eddy momentum flux at that latitude, and latitudes for which $>50 \%$ of the vectors are outside the range are completely excluded from the analysis. repeated the tracking of the $42^{\circ} \mathrm{N}$ eastward jet region in Rev 52 images using target boxes of latitudinal width $0.5^{\circ}$, which gives us better latitudinal resolution but was not selected for general use because it produces more false correlation matches (Fig. 4, upper panel). Spatial resolution at the pixel level is also degraded at times when we are required to perform $2 \times 2$ pixel summing. We therefore re-tracked the same set of images (which were not pixel-summed) with $2 \times 2$ pixel summing imposed (Fig. 4 , lower panel). Neither the change in latitudinal width of the target box nor the degradation of pixel resolution had any significant effect on our estimate of wind speed at the jet core. Finally, we performed another test in which the radius of Saturn in the navigation step was increased by $150 \mathrm{~km}$ for MT2 images; this had virtually no impact on our results. We are therefore confident that vertical variations in the latitudinal structure of winds and eddy momentum fluxes that we present later are real and not artifacts of the tracking procedure.

With the additional quality control imposed, our final dataset includes 96,394 CB2 wind vectors (48\% of the total wind vector population before any quality criteria are imposed). Thus, the Saturn dataset is now slightly larger than the best-sampled Jupiter dataset compiled from the Cassini Jupiter flyby (Salyk et al., 2006) and two orders of magnitude larger than the Voyager manual tracking dataset for Saturn (Ingersoll et al., 1984). Our final MT2 automated tracking dataset contains 23,409 wind vectors (33\% of the population before quality controls are imposed), significantly smaller than the CB2 dataset because of the lower feature contrast in MT2 images but still twice as large as the CB2 dataset used for our initial Cassini Saturn wind analysis (Del Genio et al., 2007).

Since consecutive target boxes are separated by $0.5^{\circ}$, less than the $2^{\circ}$ width of each box, the number of independent vectors is fewer than the actual number of vectors. To estimate this, we chose 12 latitudes, 6 each in cyclonic and anticyclonic shear zones (locations in which we anticipate non-negligible eddy momentum fluxes). For each of six image mosaics at these latitudes during Rev 52, we correlated each target box with the three successive target boxes that partly overlap it. The resulting mean correlation
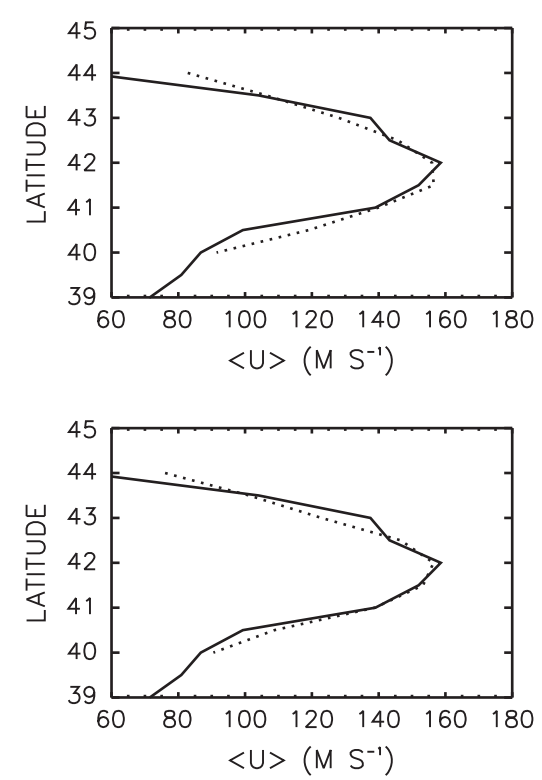

Fig. 4. Sensitivity tests for estimates of the mean zonal wind speed in the vicinity of the $42^{\circ} \mathrm{N}$ eastward jet. The solid curve in both panels shows the wind profile using the baseline algorithm on Rev 52 images. The dotted lines show the analogous wind profiles obtained when the size of the tracking box is reduced to $2^{\circ} \times 0.5^{\circ}$ (upper) and when the original resolution images are $2 \times 2$ pixel summed (lower). 
coefficients are $0.50,0.31$, and 0.25 for $0.5^{\circ}, 1.0^{\circ}$ and $1.5^{\circ}$ separation, respectively. Since we only include vectors with correlations $>0.5$ in our dataset, only the closest can be considered not to be independent of the overlapping target box. We therefore estimate that the number of independent wind estimates at each latitude is a factor of $\sim 2$ less than the total number.

Del Genio et al. (2007) discuss the contributions of pixel resolution, navigation uncertainty, and feature morphology and shape changes over one planet rotation to the total uncertainty in our derived zonal mean wind speed. The type and behavior of the tracked feature is by far the biggest contribution to the total error. Since the impact of a shape change is a decrease in the correlation coefficient within a tracking box between the first and second image, one way to estimate this error is to test the sensitivity to our correlation threshold. We therefore re-calculated the mean zonal wind for only those vectors with a correlation coefficient $>0.8$. (Note that high correlation is not synonymous with greater accuracy - wind errors due to shape changes will show up as lower correlations, whereas wind errors due to the ambiguity of repeatable linear features may manifest themselves as high correlations.) In the northern hemisphere the largest uncertainties are $\sim 3 \mathrm{~m} \mathrm{~s}^{-1}$ in the $42^{\circ} \mathrm{N}$ eastward jet and $\sim 1 \mathrm{~m} \mathrm{~s}^{-1}$ or less elsewhere. In the southern hemisphere, the poorer seeing leads to larger uncertainties in the vicinity of the eastward jets $\left(4-12 \mathrm{~m} \mathrm{~s}^{-1}\right)$ but errors of $\sim 1 \mathrm{~m} \mathrm{~s}^{-1}$ elsewhere. The larger uncertainties are restricted to a few individual $0.5^{\circ}$ latitude bands; when we average the wind profiles to $1^{\circ}$ latitudinal resolution, peak eastward jet uncertainties are reduced to $2 \mathrm{~m} \mathrm{~s}^{-1}$ and $3-9 \mathrm{~m} \mathrm{~s}^{-1}$ in the northern and southern hemispheres, respectively.

Fig. 5 shows the spatial sampling of CB2 and MT2 wind vectors for the new dataset. With the additional information provided by the 2007, 2011, and 2012 images, plus the greater capability to exclude erroneous outlier vectors, we now have relatively complete CB2 longitudinal coverage at all latitudes observable with sufficient insolation and tolerable foreshortening except for several eastward jet cores in the southern hemisphere and the latitudes closest to the equator. Remaining longitudinal gaps are due to exclusion of images near the limb and terminator rather than a systematic inability to track certain regions of Saturn. MT2 coverage is
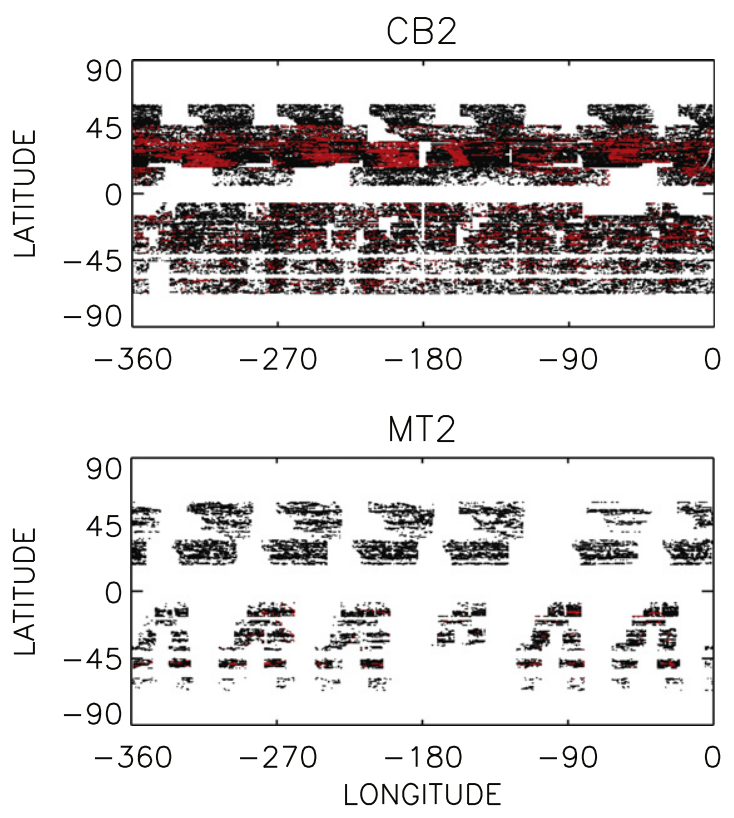

Fig. 5. Geographical distribution of automated tracking wind vectors for the (upper) CB2 and (lower) MT2 datasets. Black (red) indicate locations of single (multiple) wind vectors. more sparse because fewer images are available to track, but there is nonetheless fairly unbiased sampling in longitude. Sampling errors were not considered a major source of uncertainty in the original analysis, since we had $\sim 50-200$ wind vectors at each latitude, but any sampling errors should be approximately half as large for the new dataset since we now have $\sim 4$ times as many wind vectors per latitude.

\section{Cloud level (CB2) dynamical features}

Fig. 6 shows our complete zonal wind profile for all time periods analyzed. We calculate zonal wind in the traditional manner based on the System III estimate of Saturn's rotation period (Desch and Kaiser, 1981). In this reference frame what we refer to as "westward jets" are sometimes merely minima in eastward winds rather than actual westward wind maxima. Shorter rotation periods that imply real westward jets and weaker eastward jets have been proposed (Anderson and Schubert, 2007; Read et al., 2009b), but there is as yet no consensus on the actual internal rotation period of Saturn.

The wind profiles are generally consistent with previous Cassini estimates using different techniques within the measurement uncertainty (Porco et al., 2005; Vasavada et al., 2006; García-Melendo et al., 2011). Fig. 6 shows the García-Melendo et al. (2011) profile for comparison with ours. There are two notable significant differences: Our off-equatorial eastward jet peak of $397 \mathrm{~m} \mathrm{~s}^{-1}$ near $7.5^{\circ} \mathrm{N}$ is $10 \mathrm{~m} \mathrm{~s}^{-1}$ faster than that of García-Melendo et al. (2011), and our $157 \mathrm{~m} \mathrm{~s}^{-1}$ mean zonal wind speed in the core of the $42^{\circ} \mathrm{N}$ eastward jet is $13 \mathrm{~m} \mathrm{~s}^{-1}$ faster than their estimate.

Fig. 7 shows the CB2 mean zonal wind profile at all latitudes for which observations were available for both 2005 and 2007. The zonal wind profiles are virtually identical for the 2 years at all latitudes, differing by no more than $\sim 5 \mathrm{~m} \mathrm{~s}^{-1}$ at any latitude. The absence of time variability in the strength of the equatorial jet (admittedly over only a 2-year period) is notable given the fact that the equatorial region brightened between 2005 and 2007 from $I / F \sim 0.63$ to $\sim 0.74$ (not shown). Since there appears to be nonnegligible vertical wind shear in this region (see Section 4 ), the fact that the CB2 level wind speed is invariant during this time suggests that the brightening may be a signature of a thickening upper tropospheric haze that is not sufficient to obscure the visible cloud level, as opposed to an outbreak of deeper convective clouds as

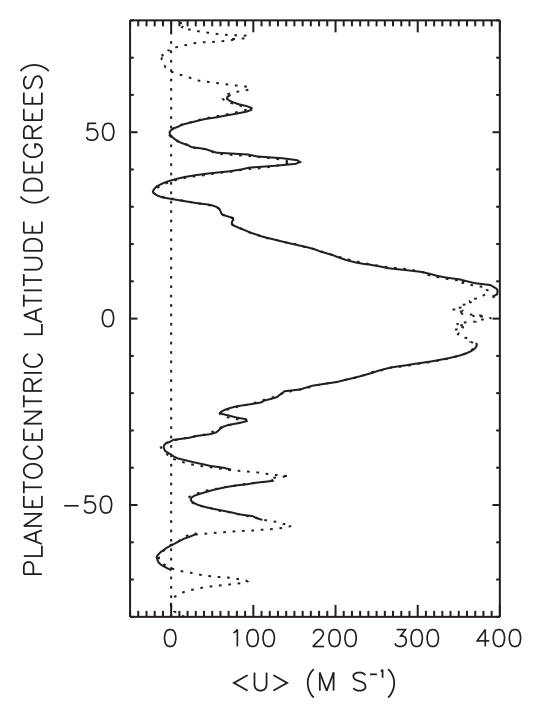

Fig. 6. Complete mean zonal wind profile for all analyzed data from this study (solid curve) vs. that of García-Melendo et al. (2011) (dotted curve). 


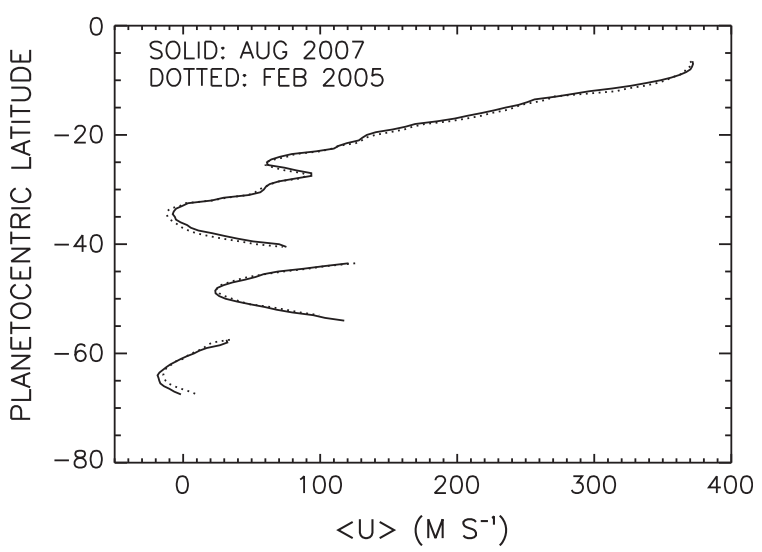

Fig. 7. Southern hemisphere mean zonal wind profiles for 2007 (solid) and 2005 (dotted).

occurred between the Voyager and Cassini eras (Porco et al., 2005). Li et al. (2011) report a $\sim 20 \mathrm{~m} \mathrm{~s}^{-1}$ strengthening of the equatorial jet near the tropopause ( $\sim 60$ mbar) between 2004 and 2008. This is not inconsistent with our result; the cloud level we observe in CB2 images is at a significantly higher pressure and thus (a) has a much longer radiative relaxation time than the tropopause, and (b) is less affected by any transient wave activity that occurs in the stratosphere.

The left panel of Fig. 8 shows the latitudinal profile of mean zonal wind compiled from all observations analyzed in 2005, 2007, 2011, and 2012. In the northern hemisphere our coverage terminates at high latitudes due to low insolation, foreshortening, and image extent. Coverage terminates at low latitudes due to ring shadows, obscuration, and very close to the equator due to a lack of feature contrast. Otherwise, latitudinal sampling is complete. In the southern hemisphere our coverage has expanded relative to that in Del Genio et al. (2007), especially for the equatorial jet, due to the additional quality control included in the tracking algorithm. However we are still unable to successfully track all the way to the cores of several higher latitude eastward jets, according to the objective criteria described in the previous section.

The middle panel of Fig. 8 shows the latitudinal profile of the zonal mean eddy momentum flux $\left\langle u^{\prime} v^{\prime}\right\rangle$, where $u$ and $v$ are the local zonal and meridional wind, respectively, \langle\rangle represents the zonal average, and the primes represent the eddy (local deviations from the zonal mean) wind components $u^{\prime}=u-\langle\mathbf{u}\rangle$ and $v^{\prime}=v-\langle v\rangle$. The eddy flux tends to be near zero near the cores of the jets and in general is directed equatorward in cyclonic shear regions and poleward in anti-cyclonic regions. In other words, the eddy flux is directed into the cores of eastward jets on both flanks, accelerating them $\left(\partial\left\langle u^{\prime} v^{\prime}\right\rangle / \partial y<0\right.$, where $y$ is latitude), and away from the westward jet cores, decelerating them $\left(\partial\left\langle u^{\prime} v^{\prime}\right\rangle / \partial y>0\right)$. The eddy flux pattern is noisy, but the automated tracking algorithm ensures fairly uniform sampling (Fig. 5, upper panel); at almost all latitudes the eddy flux estimate is based on at least 200 samples and sometimes as many as 800-900 samples (Fig. 8, right panel).

Fig. 8 also shows the first observations of eddy momentum flux for Saturn's equatorial region. It behaves similarly to other jets, with equatorward, though weak, eddy momentum fluxes at most latitudes on both its northern and southern flanks but strong equatorward eddy momentum fluxes just outside $10^{\circ}$ latitude in both hemispheres. The larger magnitude of the $\left\langle u^{\prime} v^{\prime}\right\rangle$ peak on the northern flank of the equatorial region relative to that on the southern flank might be an artifact of the lower resolution WACs used to image the northern flank, but it might also be real considering that the off-equatorial jet mean zonal wind peak is stronger on the northern side than the southern side as well. There is also a hint of a transition to poleward eddy momentum fluxes closer to the equator on the northern side, though our tracking technique begins
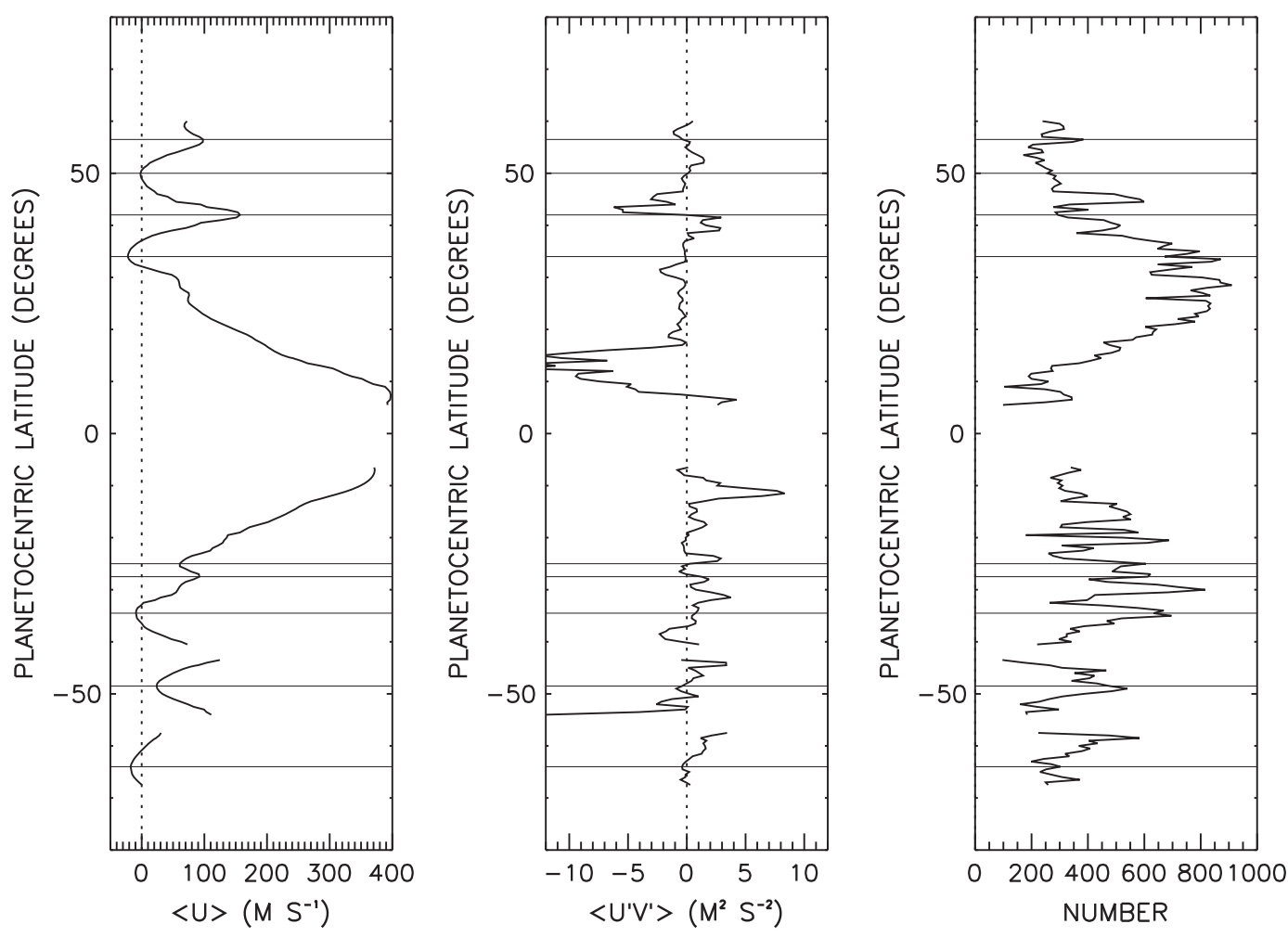

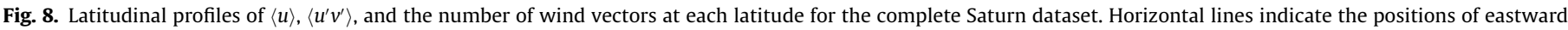
and westward jets. The $\left\langle u^{\prime} v^{\prime}\right\rangle$ curve has been smoothed to $1^{\circ}$ latitudinal resolution. 


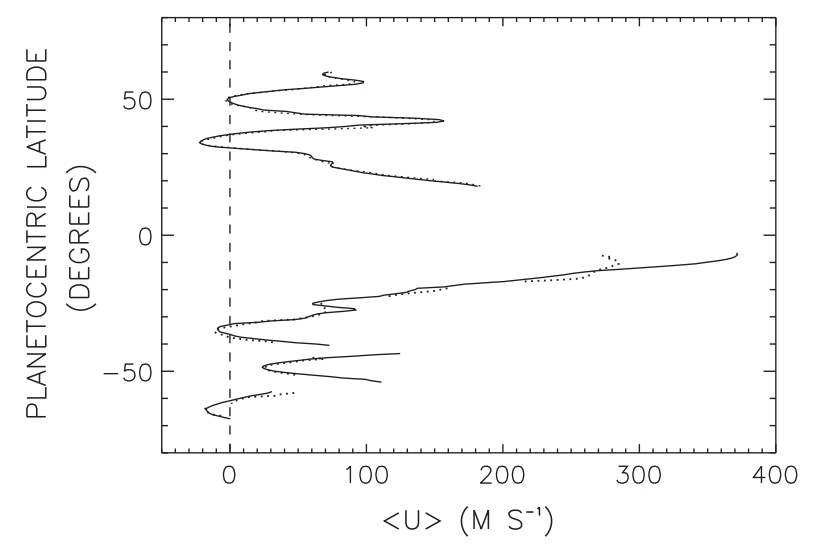

Fig. 9. Zonal mean profiles of zonal wind from CB2 (solid) and MT2 (dotted) images for all latitudes at which winds were tracked in both filters.

to fail here and thus this feature must be regarded as uncertain. In both hemispheres, the equatorial jet gives way at higher latitudes to a secondary weak jet at $\sim 27^{\circ}$ latitude, but this feature is not associated with a switch to poleward eddy fluxes. The first poleward fluxes are not seen until $\sim 35^{\circ}$ latitude in both hemispheres, where the first true westward jets in System III occur and where large-scale, deep, long-lived mesoscale convective clusters have been observed during the Cassini era (Porco et al., 2005; Sánchez-Lavega et al., 2011; Sayanagi et al., 2011).

\section{Upper troposphere (MT2) dynamical features}

Fig. 9 shows the latitudinal profile of mean zonal wind from MT2 images superimposed upon the CB2 profile. With the automated tracking algorithm we are able to compute MT2 winds at all latitudes that we observe in the northern hemisphere; in the southern hemisphere, we are less able to track in the eastward jet cores than is possible in CB2 images because of the generally lower feature contrast and greater linearity of features in MT2 images (Fig. 1). The most obvious difference between CB2 and MT2 is in the equatorial region, where winds at the MT2 level are $\sim 100 \mathrm{~m} \mathrm{~s}^{-1}$ weaker than at the CB2 level, consistent with previous estimates for the Cassini era (Porco et al., 2005; GarcíaMelendo et al., 2011).

Otherwise, zonal winds are similar to within several $\mathrm{m} \mathrm{s}^{-1}$ in CB2 and MT2, except for the slightly slower MT2 winds that can be seen in all eastward jet cores. To make this behavior clearer, we created composite wind profiles for three eastward jet and four westward jet regions (Fig. 10, left panels). The eastward jet composite (upper left) indicates that zonal winds at the core are $\sim 10 \mathrm{~m} \mathrm{~s}^{-1}$ slower at the MT2 level than at the CB2 level. This difference is larger than our uncertainty estimate and consistent with (though perhaps a bit smaller than) previous estimates based on an ISS image line-shifting technique (García-Melendo et al., 2011) and a CIRS thermal wind calculation (Fletcher et al., 2008).

On the flanks of the eastward jets, the difference reverses sign, with MT2 level winds being $\sim 5-8 \mathrm{~m} \mathrm{~s}^{-1}$ stronger than CB2 level winds. We are less confident of this feature, since the difference is smaller and because the wind speed estimate on the flanks of the $42^{\circ} \mathrm{N}$ jet is slightly sensitive to image and tracking box resolution (Fig. 4). However, other evidence suggests that this aspect of the wind profile structure is real. The upper right panel of Fig. 10 shows eastward jet composite profiles of eddy momentum flux, including the first estimate of the flux ever made at MT2 levels. The CB2 composite eddy flux, as noted earlier, is into the jet core on both flanks. The MT2 eddy flux exhibits the same behavior but with weaker magnitude, i.e., the eddy momentum flux convergence that accelerates the jet appears to weaken from the visible cloud level to the upper troposphere. If the apparent strengthening of the wind with altitude on the jet flanks is real, this implies that the eastward jets broaden with height, exactly the behavior that would be expected if the eddy momentum flux convergence weakens with altitude (e.g., see the review by Dritschel and McIntyre (2008)). Note that the equatorial jet (which is not included in the Fig. 10 composites) also exhibits clearly stronger winds at the MT2 level relative to the CB2 level on its southern flank (Fig. 9; see also García-Melendo et al., 2011).

The behavior of the westward jets is quite different. The composite mean zonal wind profile (Fig. 10, lower left) is the same in the CB2 and MT2 images to within our ability to measure them (consistent with the previous estimates by Fletcher et al. (2008) and García-Melendo et al. (2011)), and the composite MT2 eddy momentum flux profile, though noisier than its CB2 counterpart, has a similar shape and magnitude (Fig. 10, lower right).

Ingersoll et al. (1981) estimate the error in $\left\langle u^{\prime} v^{\prime}\right\rangle$ calculations from cloud tracked winds as $\sim \delta u \sigma_{v} / N^{1 / 2}$, where $\delta u$ is the root mean square zonal wind speed deviation from the mean, $\sigma_{v}$ is the uncertainty in the meridional wind, and $N$ is the number of independent wind vectors at each latitude. In our data $\delta u \sim 5-10 \mathrm{~m} \mathrm{~s}^{-1}$ (see Fig. 4 of Del Genio et al. (2007)). Uncertainties due to changes in the shapes of cloud features can in principle be large, but our correlation threshold criterion eliminates the worst cases and our other quality control criteria eliminate others. Both we and Sromovsky et al. (1983) find that the variance in meridional wind is much smaller than that for zonal wind on Saturn. We take $\sigma_{v} \sim 1-2 \mathrm{~m} \mathrm{~s}^{-1}$ as a reasonable upper limit for the uncertainty. At most latitudes we obtain 300-600 CB2 wind vectors (Fig. 8). The number of independent vectors is a factor of $\sim 2$ less because of the overlap of target boxes discussed earlier, giving $N \sim 150-300$. The Ingersoll et al. (1981) formula then yields a typical $\left\langle u^{\prime} v^{\prime}\right\rangle$ error of $\sim 0.75 \mathrm{~m}^{2} \mathrm{~s}^{-2}$ at any latitude. Our MT2 population is $\sim 25 \%$ as large as the CB2 population, so the error in $\left\langle u^{\prime} v^{\prime}\right\rangle$ at MT2 altitudes is about twice as large, $\sim 1.5 \mathrm{~m}^{2} \mathrm{~s}^{-2}$. Finally, since the $\left\langle u^{\prime} v^{\prime}\right\rangle$ profiles in Fig. 10 are composited over either 3 or 4 jets, the uncertainty in the composites is further reduced by a factor of 1.7 or 2 . We therefore conclude that the MT2 eddy fluxes, though small, are real and different from the CB2 eddy fluxes in the eastward jets, especially given their consistency in sign at various latitudes on the same side of the jet peak.

The product $\left\langle u^{\prime} v^{\prime}\right\rangle \partial\langle u\rangle / \partial y$ represents the rate of eddy to zonal kinetic energy conversion per unit mass and is potentially diagnostic of the physical processes maintaining the jets. Fig. 11 (upper left) shows that $\left\langle u^{\prime} v^{\prime}\right\rangle$ and $\partial\langle u\rangle / \partial y$ are positively correlated globally at the CB2 cloud level, confirming that eddy energy plays a role in driving the jets. The (nearly) global mean value of $4.1 \times$ $10^{-5} \mathrm{~m}^{2} \mathrm{~s}^{-3}$ is somewhat larger than our initial estimate (Del Genio et al., 2007) and about half the magnitude estimated by Salyk et al. (2006) for Jupiter. The increase appears to be the result of the addition of latitudes of strong eddy flux on the north equatorial jet flank, but partially offset by rejection of some large eddy flux values by the more stringent quality control criteria used here. The energy conversion rate is larger than the global mean value in the extratropical eastward jet regions (Fig. 11, lower left) but less than half as large in the westward jet regions (Fig. 11, lower right). The global mean conversion rate is weaker at the upper troposphere level sensed by MT2 (Fig. 11, upper right), although the difference is exaggerated since we do not have MT2 tracking of the complete north equatorial jet flank. The weaker MT2 conversion rate may partly reflect the greater difficulty in measuring winds in the MT2 images, but it is at least qualitatively realistic considering the systematically weaker latitudinal wind shear and eddy momentum fluxes in the eastward jets at MT2 levels (Fig. 10). 

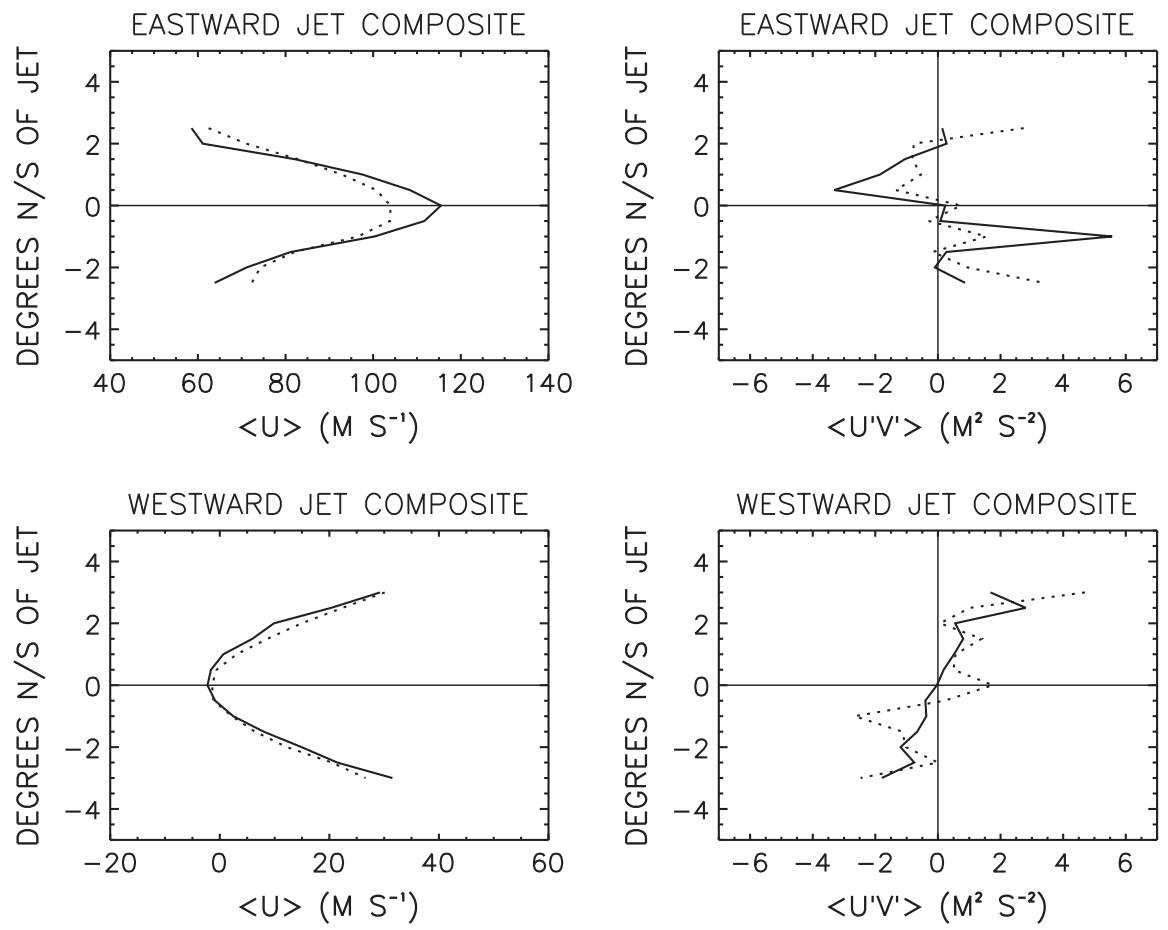

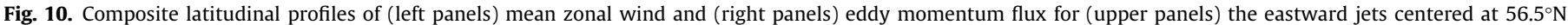

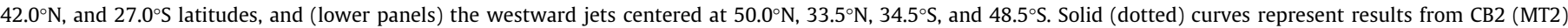
images.
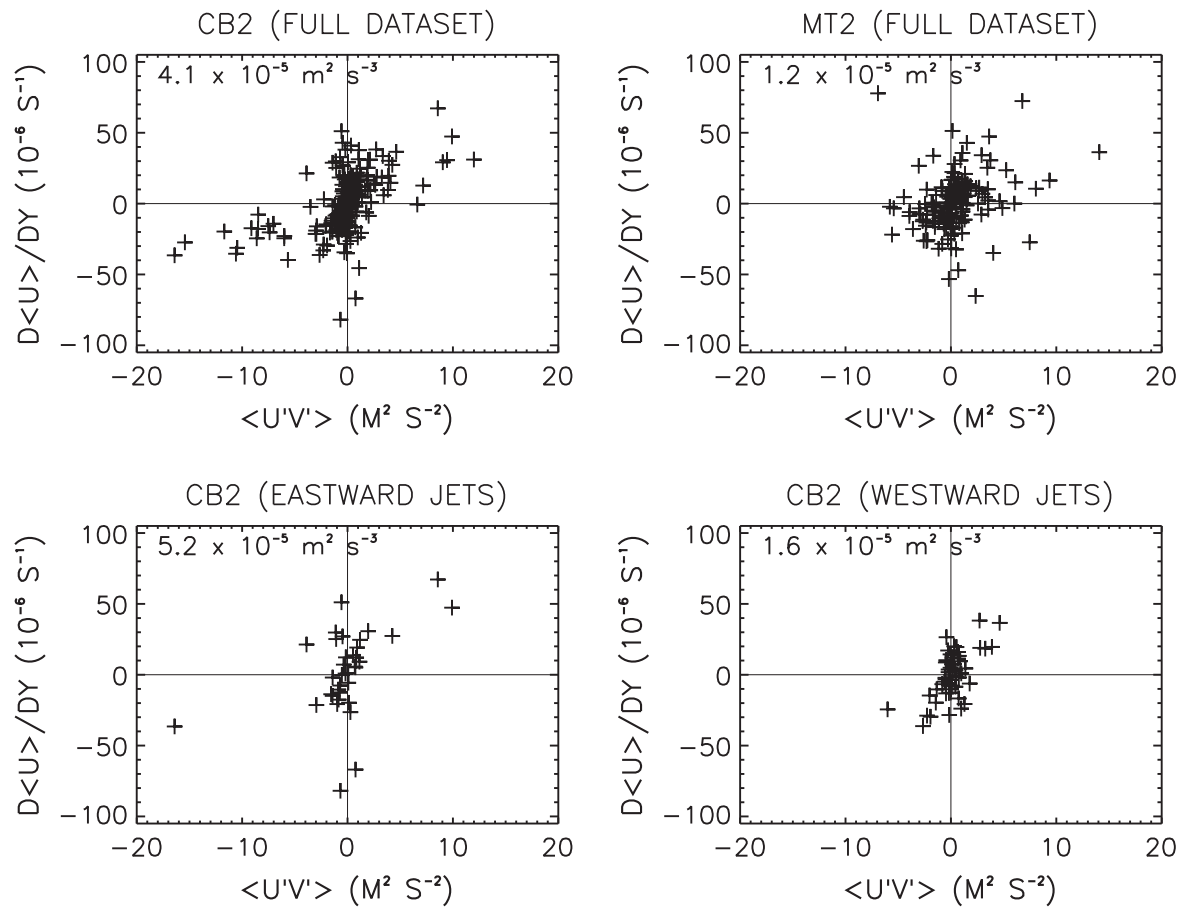

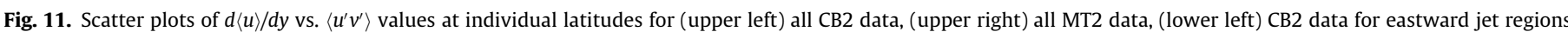

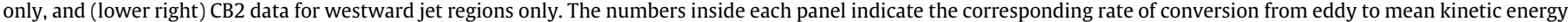
for each region.

\section{Discussion}

The first-order questions about the dynamics of Saturn's atmosphere are whether the mean zonal flow long observed at cloud tops is shallow or deep, and whether the processes driving it are themselves shallow or deep. An extensive discussion of the candidate theories can be found in Del Genio et al. (2009). Shallow "weather layer" models of the flow rely on forcing by radiative heating due to differential insolation or latent heat release due to the condensation of water, while deep flow configurations regard 
the cloud level winds as the surface manifestation of deep convective cylinders forced by Saturn's internal heat source. None of the proposed models can be regarded as conclusive for three reasons: (1) There is no direct observational evidence of the dynamical conditions deep in Saturn's atmosphere; (2) Modeling of the effects of moist convection has thus far been highly idealized; (3) Computational limitations have made it difficult to simulate the full 3-dimensional dynamics of large, deep jovian planet atmospheres without making simplifying assumptions and/or using unrealistically strong forcing and drag. Furthermore, a combination of forcing mechanisms may be relevant, e.g., with different physics producing the equatorial jet and the alternating extratropical jets.

The results presented here provide several new observational constraints that can be used to evaluate potential forcing mechanisms and flow configurations. Del Genio et al. (2007) hypothesized that the eddy momentum fluxes that are observed to converge at Saturn's eastward jets and diverge at Saturn's westward jets (similar to behavior observed for Jupiter by Salyk et al. (2006)) explain the maintenance of the jets. The vertical structure we have now observed provides additional evidence for this: Eastward jets are sharp at the CB2 cloud level but weaken and broaden in the upper troposphere where MT2 sees. Consistent with this, eddy momentum fluxes (and flux convergences) decrease upward in the eastward jet regions (Fig. 10).

Recent shallow weather layer models driven by condensation heating (e.g., Lian and Showman, 2010) and by differential insolation (e.g., Liu and Schneider, 2010) both produce horizontal eddy momentum flux patterns consistent with what we observe. They also simulate the "staircase" profile of potential vorticity inferred by Read et al. (2009a) from Cassini CIRS upper troposphere temperatures and Voyager/Cassini cloud level winds. However, the fact that the eddy fluxes we observe weaken upward suggests that the process responsible for them is driven at or below the cloud level rather than above. Pérez-Hoyos and Sánchez-Lavega (2006) estimate that most of the incident solar flux on Saturn is absorbed in the upper troposphere haze and that little penetrates below the 600 mbar level. By itself, radiative heating in the upper troposphere that leads to eddy forcing that drives mean overturning circulations and jets at deeper levels via the "downward control" principle (Haynes et al., 1991) is perfectly plausible. However, we would expect the eddy-induced forcing per unit mass $\mathcal{F}$ to be a maximum within or above the level at which the heating occurs. Instead, our results suggest that $\mathcal{F}$ (i.e., $-\partial\left\langle u^{\prime} v^{\prime}\right\rangle / \partial y$ ) is small within the layer of radiative heating and stronger below. Thus for Saturn at least, radiative driving of the extratropical jets via baroclinic instability, as proposed by Liu and Schneider (2010) (who assume an optical thickness of only 3 down to the 3 bar lower boundary of their model), appears to be inconsistent with our data.

Choi et al. (2009) have derived zonal winds at a depth of several bars from tracking emission features in Cassini VIMS $5 \mu \mathrm{m}$ images. There is too much scatter in their results to detect whether a subtle vertical shear exists between that level and the CB2 level, but a first-order decay or strengthening of the extratropical jets over $\sim 1-2$ bars below the levels seen by ISS can be ruled out. Nothing can be said about decay or strengthening of the jets below the level that VIMS senses.

No direct evidence about eddy momentum fluxes exists below the CB2 cloud level, but our estimated global mean eddy-zonal kinetic energy conversion rate per unit mass implies a conversion rate of $\sim 0.25 \mathrm{~W} \mathrm{~m}^{-2}$ per bar of atmosphere through which the eddy fluxes penetrate. As Liu and Schneider (2010) point out, this is already $\sim 10 \%$ of the Saturn internal heat flux, and given the low efficiency with which atmospheric circulations convert potential into kinetic energy, it is unlikely that these eddy fluxes penetrate much deeper than the level that ISS observes. Anelastic models of deep cylindrical convection (Kaspi et al., 2009) produce large eddy momentum fluxes at depth when forced with excessive internal heat fluxes; it remains to be seen whether such a model run with realistic forcing can restrict large horizontal eddy flux convergences and energy conversions to a shallow weather layer. This question of the forcing of the jets is distinct from the question of the depth of the jets themselves (e.g., Showman et al., 2006) the jets may well be deep, but the eddy forcing that we observe probably does not penetrate deeply. Other potential contributions to jet forcing that might originate at depth, e.g., vertical eddy momentum fluxes, cannot be constrained by our analysis.

It has often been noted that westward jets on the jovian planets violate the classical Rayleigh-Kuo criterion for barotropic instability, suggesting that a different criterion may be relevant for a three-dimensional fluid (see the discussion in Read et al. (2009a)). The broad shape of the westward jets (Fig. 10), and the relatively flat profile of potential vorticity across them, might be construed as evidence of mixing by barotropic instability (although Read et al. note that in some of these jets there is actually a potential vorticity gradient reversal). Barotropic instability converts zonal kinetic energy to eddy kinetic energy, resulting in a negative energy conversion rate, the opposite of what we observe in the westward jets (Fig. 11, lower right). Episodic barotropic eddy generation might nonetheless occur and regulate the shape of the westward jets. However, the largest energy conversion rates we see are in the sharper eastward jets (Fig. 11, lower left). It is therefore natural to look to these locations for the driving process.

One of the strongest and most discussed eastward jets on Saturn is at $42^{\circ} \mathrm{N}$, the location of the so-called "ribbon wave" (Fig. 12). Godfrey and Moore (1986) proposed that the ribbon was a manifestation of baroclinic instability of the eastward jet. This is consistent with the strong eddy momentum flux convergence into the jet that we see at this latitude (Fig. 8), associated with the small-scale chevron pattern clearly visible in Fig. 12, and the sharp gradient in potential vorticity at the jet latitude observed by Read et al. (2009a). The ribbon is a bright, distinct feature in CB2 images but is at least weakly visible at some longitudes at MT2 levels (Fig. 12), suggesting that it is a vertically coherent phenomenon. Fletcher et al. (2010) show that middle and upper troposphere temperatures on either side of the ribbon latitude have been fairly variable during the Cassini mission. Sayanagi et al. (2010) modeled the jet and found that it becomes unstable and produces a propagating ribbon-like feature when a potential vorticity reversal occurs. However, in their model the wave propagates westward relative to the mean flow at a substantial phase speed, in disagreement with Voyager data (Sromovsky et al., 1983).

Our peak jet speed of $157 \mathrm{~m} \mathrm{~s}^{-1}$ is $13 \mathrm{~m} \mathrm{~s}^{-1}$ faster than that measured by García-Melendo et al. (2011), who used a one-dimensional line-shifting correlation technique for $50-100^{\circ}$ longitudinal segments. It is tempting to speculate that the difference between their estimate and ours represents the westward phase speed of the wave relative to the flow, since the line-shifting approach may detect large-scale periodic features. The probability density function (pdf) of wind speed from our tracking algorithm at $42^{\circ} \mathrm{N}$ is shown in Fig. 13; it is intermediate between the best and worst case scenarios shown in Fig. 3. There is a sharply defined mode value of $160 \mathrm{~m} \mathrm{~s}^{-1}$, decreasing to zero occurrence at $178 \mathrm{~m} \mathrm{~s}^{-1}$. This defines the two-sided range of wind speed values that we include in calculating the mean according to the $1 \%$ of the mode value cutoff criterion described in Section 2. Below $160 \mathrm{~m} \mathrm{~s}^{-1}$ the pdf drops off more slowly, with a long flat tail that extends down to $38 \mathrm{~m} \mathrm{~s}^{-1}$. This randomly distributed behavior is characteristic of false identifications by automated feature tracking algorithms in regions of linear or periodic feature morphology (Rossow et al., 1990).

We divided this apparently random part of the distribution into two segments, with speeds $>$ or $<106 \mathrm{~m} \mathrm{~s}^{-1}$, where the first obvious minimum in the population below the symmetric $1 \%$ test cutoff 

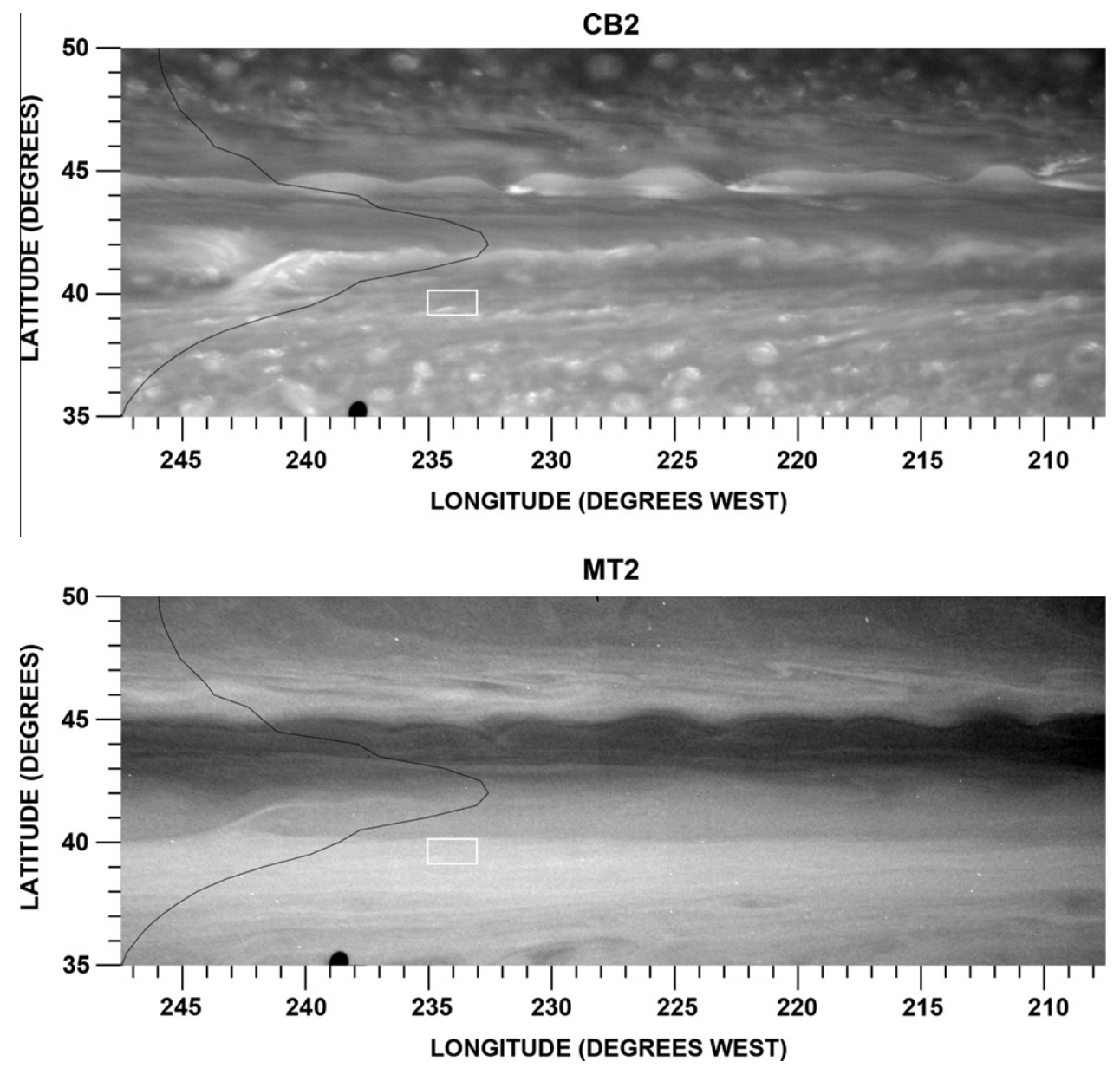

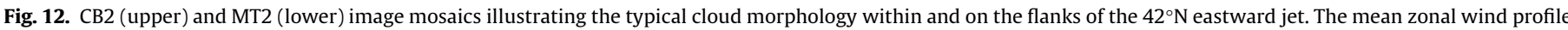
is shown at the left. The box highlights a feature at $39.5^{\circ} \mathrm{N}, 234^{\circ} \mathrm{W}$ that was estimated to have a value of $u^{\prime} v^{\prime}=6.4 \mathrm{~m}^{2} \mathrm{~s}^{-2}$ at the $\mathrm{CB} 2$ level.

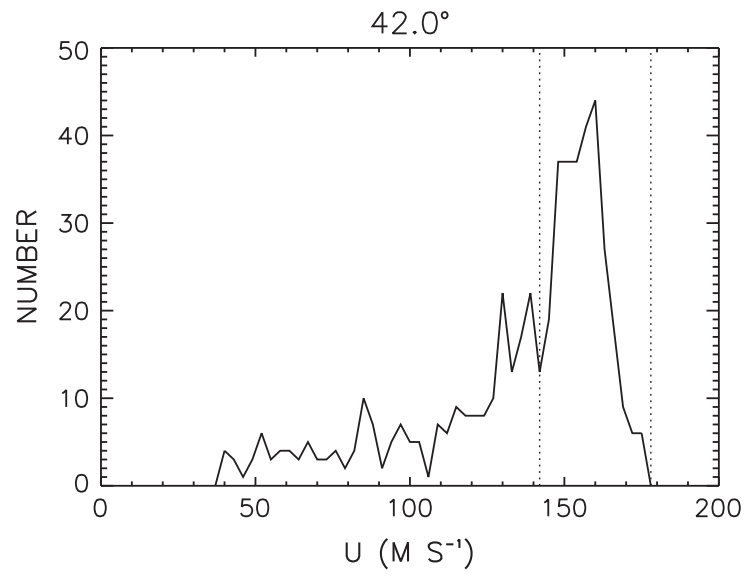

Fig. 13. As in Fig. 3 but for the $42^{\circ} \mathrm{N}$ eastward jet at the latitude of the Saturn ribbon wave.

point occurs. Of 10 randomly selected vectors between 38-106 $\mathrm{m} \mathrm{s}^{-1}, 8$ were found to be spurious and 2 ambiguous identifications (i.e., examples that would not be chosen by manual tracking) upon visual inspection. If only wind speeds below $106 \mathrm{~m} \mathrm{~s}^{-1}$ are excluded, the mean wind speed is $148 \mathrm{~m} \mathrm{~s}^{-1}$. We also inspected 10 randomly selected vectors between 106 and $142 \mathrm{~m} \mathrm{~s}^{-1}$; two were clearly spurious, the others ambiguous. Finally, of 10 randomly selected vectors between 142 and $178 \mathrm{~m} \mathrm{~s}^{-1}$ (the population retained for the actual mean wind estimate), 7 were accurate and 3 ambiguous, supporting our original estimate of a $157 \mathrm{~m} \mathrm{~s}^{-1}$ mean wind at the jet core.

Finally, we re-tracked the wind at $42.0^{\circ} \mathrm{N}$ using tracking boxes that were either 1 or 10 pixels wide in latitude and $20^{\circ}$ in longitude to mimic the line-shifting technique in our 12 available image pairs at this latitude. The resulting wind speed estimates range from 113 to $174 \mathrm{~m} \mathrm{~s}^{-1}$, most with low correlation coefficients. The two highest correlation $(0.78,0.93)$ targets give speeds of 132 and $135 \mathrm{~m} \mathrm{~s}^{-1}$ (for the same image pair but with different latitudinal widths of the tracking box). However the source of these wind estimates is a segment dominated by the diffuse bright feature near $245^{\circ}$ longitude in Fig. 12 rather than by any wavelike feature. Segments with more periodic morphologies instead track with speeds of $\sim 167 \mathrm{~m} \mathrm{~s}^{-1}$. Thus the reason for our faster jet speed relative to that of García-Melendo et al. (2011) remains unclear.

If baroclinic instability occurs in the eastward jets, the most plausible source appears to be horizontal temperature gradients associated with latent heat release, which we would anticipate to exist near or below the CB2 cloud level. Lian and Showman (2010) have had some success in generating extratropical jets in this way, using a model with a simplified hydrological cycle that simply condenses and precipitates supersaturated vapor at the grid scale and replenishes the vapor from an assumed deep reservoir. On Saturn, latent heat release is more likely due to deep convection. Convective latent heat release may be more sporadic and will almost certainly have a different vertical heating profile than gridscale condensation. Liu and Schneider (2010) argue that realistic moist convective driving requires a latent heat flux from the interior that is consistent with the internal heat source. Nonetheless, 
the observed distribution of convective clouds on Saturn, with a concentration in cyclonic regions and an absence in anti-cyclonic regions (Del Genio et al., 2007) is at least consistent with the idea that convective latent heat release might create the lateral temperature gradients required to drive baroclinic instability.

It is also conceivable that baroclinic instability is not involved at all. Dritschel and McIntyre (2008) suggest that upward-propagating Rossby waves forced by convective motions from the deep interior could propagate along the eastward jet waveguide and produce the observed potential vorticity mixing pattern on either side. Convective forcing from below does generate Rossby waves at the equator in several Saturn numerical models (Sayanagi and Showman, 2007; Liu and Schneider, 2010, 2011). In the Liu-Schneider experiments, the resulting equatorward eddy momentum fluxes (consistent with what we observe; Fig. 8) create a prograde equatorial jet when the effect of the internal heat flux outweighs that of baroclinicity. Furthermore, the observed off-equatorial peaks in the wind speed of the equatorial jet on both Saturn (Fig. 6) and Jupiter (Porco et al., 2003) are consistent with strong potential vorticity mixing in a low Richardson number environment (Allison et al., 1995), such as might be produced by convective Rossby waves. In the extratropics, though, where heating is not simply balanced by divergence, it is not clear whether such a mechanism can be as effective.

Our results continue to imply that the Saturn mean circulation at the CB2 cloud level is an indirect eddy-driven cell with rising motion in the cyclonic shear regions, equatorward flow aloft across eastward jets, and sinking in the anti-cyclonic shear regions (Del Genio et al., 2007). The first-order force balance at the cloud level is thus between acceleration by the eddy momentum flux convergence and deceleration by the Coriolis torque on the equatorward mean flow. This circulation pattern is required by the observed preference of moist convection for cyclonic regions. This preference in turn can be explained by the requirement that the return flow at depth converge moisture in the cyclonic shear region (see Fig. 7.18 of Del Genio et al., 2009). Enhanced $\mathrm{NH}_{3}$ concentrations poleward of the $42^{\circ} \mathrm{N}$ jet also imply rising motion there (Fletcher et al., 2011). At higher levels, the meridional circulation may reverse (Del Genio et al., 2009; Fletcher et al., 2011).

The force balance at depth on the jovian planets is unknown. Strong mean zonal winds might extend down to the water condensation level or even deeper, despite being forced at cloud level, as a result of "downward control" (Haynes et al., 1991; Showman et al., 2006). One idea is that the Coriolis acceleration due to the poleward mean flow in the deep return branch of the eddy-driven cell is balanced by magnetohydrodynamic drag at depths where the molecular hydrogen envelope becomes more conducting (Liu and Schneider, 2010). However, other sources of dissipation not yet accounted for in jovian planet models may be important within the cloud layer itself, e.g., momentum mixing by moist convection (Gregory et al., 1997) or frictional drag by falling precipitation particles, which may be the primary source of dissipation in Earth's tropics (Pauluis and Dias, 2012). It would be of interest to see whether the inclusion of such processes in models of the jovian planets can produce a true closed shallow weather layer circulation that extends not much farther down than the water condensation level but which satisfies the observed cloud level constraints shown in this paper as well as global energetic constraints.

\section{Acknowledgments}

This research was supported by NASA Cassini Project funding of the Imaging Science Subsystem team. We thank the reviewers for constructive comments that helped improve the manuscript.

\section{References}

Allison, M., Del Genio, A.D., Zhou, W., 1995. Richardson number constraints for the Jupiter and outer planet wind regime. Geophys. Res. Lett. 22, 2957-2960.

Anderson, J.D., Schubert, G., 2007. Saturn's gravitational field, internal rotation, and interior structure. Science $317,1384-1387$

Choi, D.S., Showman, A.P., Brown, R.H., 2009. Cloud features and zonal wind measurements of Saturn's atmosphere as observed by VIMS. J. Geophys. Res. 114, E04007. http://dx.doi.org/10.1029/2008JE003254.

Del Genio, A.D., Rossow, W.B., 1990. Planetary-scale waves and the cyclic nature of cloud top dynamics on Venus. J. Atmos. Sci. 47, 293-318.

Del Genio, A.D. et al., 2007. Saturn eddy momentum fluxes and convection: First estimates from Cassini images. Icarus 189, 479-492.

Del Genio, A.D. et al., 2009. Saturn atmospheric structure and dynamics. In: Dougherty, M.K., Esposito, L.W., Krimigis, S.M. (Eds.), Saturn from CassiniHuygens. Springer, New York, pp. 113-160.

Desch, M.D., Kaiser, M.L., 1981. Voyager measurement of the rotation period of Saturn's magnetic field. Geophys. Res. Lett. 8, 253-256.

Dritschel, D.G., McIntyre, M.E., 2008. Multiple jets as PV staircases: The Phillips effect and the resilience of eddy transport. J. Atmos. Sci. 65, 855-874.

Fletcher, L.N. et al., 2008. Temperature and composition of Saturn's polar hot spots and hexagon. Science 319, 79-82.

Fletcher, L.N., Achterberg, R.K., Greathouse, T.K., Orton, G.S., Conrath, B.J., SimonMiller, A.A., Teanby, N., Guerlet, S., Irwin, P.G.J., Flasar, F.M., 2010. Seasonal change on Saturn from Cassini/CIRS observations. Icarus 208, 337-352.

Fletcher, L.N., Baines, K.H., Momary, T.W., Showman, A.P., Irwin, P.G.J., Orton, G.S., Roos-Serote, M., Merlet, C., 2011. Saturn's tropospheric composition and clouds from Cassini/VIMS 4.6-5.1 micron nightside spectroscopy. Icarus 214, 510-533.

García-Melendo, E., Sánchez-Lavega, A., Rojas, J.F., Pérez-Hoyos, S., Hueso, R., 2009. Vertical shears in Saturn's eastward jets at cloud level. Icarus 201, 818-820.

García-Melendo, E., Pérez-Hoyos, S., Sánchez-Lavega, A., Hueso, R., 2011. Saturn's zonal wind profile in 2004-2009 from Cassini ISS images and its long-term variability. Icarus 202, 181-196.

Godfrey, D.A., Moore, V., 1986. The saturnian ribbon feature - A baroclinically unstable model. Icarus 68, 313-343.

Gregory, D., Kershaw, R., Inness, P., 1997. Parameterization of momentum transport by convection - II: Tests in single column and general circulation models. Q. J. R. Meteorol. Soc. 123, 1153-1183.

Haynes, P.H., McIntyre, M.E., Shepherd, T.G., Marks, C.J., Shine, K.P., 1991. On the "downward control" of extratropical diabatic circulations by eddy-induced mean zonal forces. J. Atmos. Sci. 48, 651-678.

Heimpel, M., Aurnou, J., 2007. Turbulent convection in rapidly rotating spherical shells: A model for equatorial and high latitude jets on Jupiter and Saturn. Icarus 187, 54-557.

Ingersoll, A.P., Beebe, R.F., Mitchell, J.L., Garneau, G.W., Yagi, G.M., Shu, F.H., 1981 Interaction of eddies and zonal flow on Jupiter as inferred from Voyager 1 and 2 images. J. Geophys. Res. 86 (A10), 8733-8743.

Ingersoll, A.P., Beebe, R.F., Conrath, B.J., Hunt, G.E., 1984. Structure and dynamics of Saturn's atmosphere. In: Gehrels, T., Mathews, M.S. (Eds.), Saturn. University of Arizona Press, Tucson, pp. 195-238.

Karkoschka, E., Tomasko, M., 2005. Saturn's vertical and latitudinal cloud structure 1991-2004 from HST imaging in 30 filters. Icarus 179, 195-221.

Kaspi, Y., Flierl, G.R., Showman, A.P., 2009. The deep wind structure of the giant planets: Results from an anelastic general circulation model. Icarus 202, 525542

Li, L. et al., 2011. Equatorial winds on Saturn and the stratospheric oscillation. Nat. Geosci. 4, 750-752.

Lian, Y., Showman, A.P., 2010. Generation of equatorial jets by large-scale latent heating on the giant planets. Icarus 207, 373-393.

Liu, J., Schneider, T., 2010. Mechanisms of jet formation on the giant planets. J. Atmos. Sci. 67, 3652-3672.

Liu, J., Schneider, T., 2011. Convective generation of equatorial superrotation in planetary atmospheres. J. Atmos. Sci. 68, 2742-2756.

Pauluis, O., Dias, J., 2012. Satellite estimates of precipitation-induced dissipation in the atmosphere. Science 335, 953-956.

Pérez-Hoyos, S., Sánchez-Lavega, A., 2006. Solar flux in Saturn's atmosphere: Penetration and heating rates in the aerosol and cloud layers. Icarus 180, 368378.

Pérez-Hoyos, S., Sánchez-Lavega, A., French, R.G., Rojas, J.F., 2005. Saturn's cloud structure and temporal evolution from ten years of Hubble Space Telescope images (1994-2003). Icarus 176, 155-174.

Porco, C.C. et al., 2003. Cassini Imaging Science at Jupiter. Science 299, 1541-1547.

Porco, C.C. et al., 2004. Cassini Imaging Science: Instrument characteristics and capabilities and anticipated scientific investigations at Saturn. Space Sci. Rev. $115,363-497$

Porco, C.C. et al., 2005. Cassini Imaging Science: Initial results on Saturn's atmosphere. Science 307, 1243-1247.

Read, P.L., Conrath, B.J., Fletcher, L.N., Gierasch, P.J., Simon-Miller, A.A., Zuchowski, L.C., 2009a. Mapping potential vorticity dynamics on Saturn: Zonal mean circulation from Cassini and Voyager data. Planet. Space Sci. 57, 1682-1698.

Read, P.L., Dowling, T.E., Schubert, G., 2009b. Saturn's rotation period from its atmospheric planetary-wave configuration. Nature 460, 608-610.

Rossow, W.B., Del Genio, A.D., Eichler, T.P., 1990. Cloud-tracked winds from Pioneer Venus OCPP images. J. Atmos. Sci. 47, 2053-2084. 
Salyk, C., Ingersoll, A.P., Lorre, J., Vasavada, A., Del Genio, A.D., 2006. Interaction between eddies and mean flow in Jupiter's atmosphere: Analysis of Cassini imaging data. Icarus $185,430-442$

Sánchez-Lavega, A., Hueso, R., Pérez-Hoyos, S., 2007. The three-dimensional structure of Saturn's equatorial jet at cloud level. Icarus 187, 510-519.

Sánchez-Lavega, A. et al., 2011. Deep winds beneath Saturn's upper clouds from a seasonal long-lived planetary-scale storm. Nature 475, 71-74.

Sayanagi, K.M., Showman, A.P., 2007. Effects of a large convective storm on Saturn's equatorial jet. Icarus $187,520-539$.

Sayanagi, K.M., Morales-Juberías, R., Ingersoll, A.P., 2010. Saturn's northern hemisphere ribbon: Simulations and comparison with the meandering Gulf Stream. J. Atmos. Sci. 67, 2658-2678.
Sayanagi, K.M., Dyudina, U.A., Ewald, S.P., Fischer, G., Ingersoll, A.P., Kurth, W.S. Muro, G.D., Porco, C.C., West, R.A., 2011. Dynamics of Saturn's great storm of 2010-2011 from Cassini ISS and RPWS. Icarus, submitted for publication.

Showman, A.P., Gierasch, P.J., Lian, Y., 2006. Deep zonal winds can result from shallow driving in a giant-planet atmosphere. Icarus 182, 513-526.

Sromovsky, L.A., Revercomb, H.E., Krauss, R.J., Suomi, V.E., 1983. Voyager 2 observations of Saturn's northern mid-latitude cloud features: Morphology, motions, and evolution. J. Geophys. Res. 88, 8650-8666.

Vasavada, A.R. et al., 2006. Cassini imaging of Saturn: Southern hemisphere winds and vortices. J. Geophys. Res. 111, E05004. http://dx.doi.org/10.1029/ 2005JE002563. 